for whom, consecutively, radial access was utilized during an endovascular procedure.

Results Included in our study sample are 11 total individuals found to have undergone neuroendovascular procedure, 7 of which are male and 4 are female. The mean age was 65.6 years (range from 35 to 91). 8 of the 12 patients observed required diagnostic cerebral angiograms, 2 patients required mechanical thrombectomy, and one patient underwent carotid stent placement and angioplasty for greater than $95 \%$ stenosis of the common carotid artery. The average hospital admission of this sample size was 5.2 days (range from 1 to 18). The mean National Institutes of Health Stroke Scale (NIHSS) on admission to the hospital was 5.45 (range from 0 to 28). 9 patients had scores of less than 3 on admission, while the remaining two patients has scores of 26 and 28. The mean length of procedure: for diagnostic cerebral angiograms was 25.5 minutes (range 15.2 to 56.3), for thrombectomy the mean time was 2 hours 2 minutes, and the time of stent and angioplasty was 1 hour 32 minutes. The mean fluoroscopic time for all patients was 15 minutes (range from 2.4 to 42.5). Of the 11 patients included in the sample size, 7 underwent procedures during which the treating physician was required to switch access unplanned, from femoral to radial. For 4 of the 11 patients, the treating physician planned to access the vasculature via radial access prior to the procedure. Of the 11 patients, only one experienced a complication in the form of an enlarging neck hematoma from the radial artery following percutaneous access for a cerebral angiogram. This event was treated the same day with radial artery and internal jugular repair during which hemostasis was achieved. On discharge, the mean NIHSS was 1.54 (range from 0 to 14). 10 of the 11 patients were discharged with an NIHSS of greater than or less than 2 , only one patient was discharged with a score of 14 , however, this patient was also admitted with pre-existing conditions which contributed to the higher than average access of other participants.

Conclusion In the experience of this single-center, the use of the transradial approach for neuroendovascular procedures seems to result in a relatively low rate of access complications and shifting access technique. Our results, while limited in the size of sample, contribute to the growing body of literature that sheds insight on the benefit of transradial access.

Disclosures M. Taqi: None.

\section{E-081 SUSCEPTIBILITY - WEIGHTED IMAGING FINDINGS IN EXPERIMENTAL ACUTE ISCHEMIC STROKE MODEL}

N Saadat* , G Christoforidis, M Niekrasz, S Roth, T Carroll. University of Chicago, Chicago, IL

\subsection{6/neurintsurg-2021-SNIS.176}

Purpose Assessment of the ischemic penumbra is essential in the treatment of acute ischemic stroke. This work sought to study the hyperacute changes on ischemic regions on the susceptibility-weighted imaging (SWI) using a controlled large animal experimental middle cerebral artery (MCA) occlusion model.

Methods Eleven mongrel canines $(20-30 \mathrm{~kg})$ underwent permanent endovascular occlusion of the M1 segment of MCA. Magnetic resonance imaging (MRI) was acquired on a 3T unit (Achieva, Philips Healthcare, Best, Netherlands) and included SWI and DTI starting one-hour post-MCA occlusion (MCAO). DSC perfusion MRI was subsequently acquired. Regions of

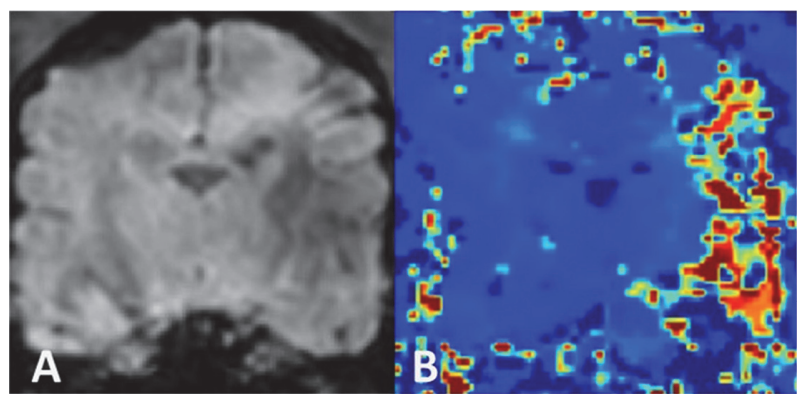

Abstract E-081 Figure 1 SWI signal (A) corresponds to elevated Tmax (B)

interest (ROIs) were manually drawn using the Image $\mathrm{J}$ software (National Institutes of Health, Bethesda, Maryland) corresponding to the MCA territory of each hemisphere based on previously reported canine anatomic studies. An independent samples t-test was conducted to compare the mean signal intensity on the MCA territory of the ischemic region and the contralateral non-ischemic region. The Mean diffusivity maps and DSC perfusion were compared to the SWI images.

Results Signal intensity was consistently lower in the ischemic territory compared to the non-ischemic territory. There was a significant difference in the mean signal intensity on the ischemic region $(\mathrm{M}=1,517.51, \mathrm{SD}=104.50)$ vs. the non-ischemic region $(\mathrm{M}=1,676.54, \mathrm{SD}=81.91) ; \mathrm{P}$-value $=0.0007$. Qualitatively, the images revealed larger cortical and medullary veins in the ischemic territory. Mean diffusivity images, cerebral blood volume, and cerebral blood flow maps did not consistently match the extent of the SWI signal loss, whereas MTT and Tmax maps consistently matched the area of signal loss on SWI.

Conclusion In the hyperacute stages of MCAO, SWI images display a consistent and significant signal drop relative to the unaffected side. This signal drop is suspected to result from the paramagnetic effect of deoxyhemoglobin and venodilation and thus an indirect means of visualizing tissue hypoxia.

Disclosures N. Saadat: None. G. Christoforidis: 1; C; NIH. M. Niekrasz: None. S. Roth: 1; C; NIH. T. Carroll: 1; C; NIH.

\section{E-082 INITIAL EXPERIENCE WITH TRANSRADIAL ACCESS FOR NEUROINTERVENTIONS: FEASIBILITY AND SAFETY OF NEXT GENERATION .088" INTRACRANIAL GUIDE CATHETERS}

L Lyons*, M Abouelleil, A Restrepo Orozco, L Verhey, J Tsai, P Mazaris, J Singer. Neurosurgery, Spectrum Health, Grand Rapids, MI

\subsection{6/neurintsurg-2021-SNIS.177}

Background Several reports have demonstrated significant clinical benefits with transradial access compared to transfemoral access for endovascular procedures. Despite this, the transradial technique for neurointerventions is underutilized due to the anatomical complexities that may affect a successful procedure using conventional access catheter technology. The purpose of this single-center study was to evaluate the feasibility and safety of next generation large-bore intracranial guide catheters (0.088" inner diameter, 8Fr) using a transradial approach (TRA) in carefully selected patients undergoing neurointerventions. 
Methods The retrospective review of the institutional, IRBapproved database was undertaken to find cases where the TracStar LDP $^{\mathrm{TM}}$ or Zoom ${ }^{\mathrm{TM}} 88$ guide catheters (Imperative Care, Campbell, CA) were used with a TRA for neurointerventions. For this study, gender, age, case type, target anatomy, distal location reached with the guide catheters, time from access to reperfusion, complications, and the Thrombolysis in Cerebral Infarction (TICI) score were collected. Safe placement of the guide catheters to the target anatomy was considered a technical success.

Results From August 2020 to March 2021, 13 patients underwent TRA neurointerventions using the TracStar LDP or Zoom 88 guide catheters. The TracStar LDP was used in 77\% $(10 / 13)$ of patients; the Zoom 88 guide catheter was used in $23 \%(3 / 13)$ of patients. The type of intervention was acute ischemic stroke in $69 \%(9 / 13)$ of patients and aneurysm embolization in $31 \%(4 / 13)$ of patients. The TracStar LDP facilitated the implantation of flow diverters in all aneurysm cases with 75\% (3/4) using a biaxial system. Overall, there was an even distribution between females $(54 \%, 7 / 13)$ and males $(46 \%, 6 / 13)$. The median age was 72.5 (range $=48-88$ ) years. Most patients with aneurysms were females (75\%, 3/4); this population's median age was 58.5 (range $=54-64$ ) years. In patients with acute ischemic stroke, the median age was 76 (range $=48-88$ ) years. The target anatomy for all aneurysm patients was the left internal carotid artery. The target anatomy for stroke patients included right internal carotid artery in $77.8 \%(7 / 9)$ of patients, left internal carotid artery in $11.1 \%(1 / 9)$ of patients and left vertebral artery in $11.1 \%$ (1/ 9) of patients. In these patients, the final positions of the guide catheters included Supraclinoid Carotid $(n=2)$, Ophthalmic segment $(n=2)$, Petrous Carotid, Petro-Cavernous junction, Basilar, Cavernous-Carotid, and in one case the Zoom 88 was positioned in the right $\mathrm{M} 1$. The median puncture to reperfusion time was 30.5 (range $=5-50$ ) minutes. The TICI $2 \mathrm{~b}$ or greater was achieved in $77.8 \%$ (7/9) of patients. There were no complications associated with the guide catheters. In two patients (one stroke, one aneurysm), the TracStar LDP guide catheter was used successfully as the rescue option after the initial approach with the 0.079 " radial guide catheter failed.

Conclusion Transradial access with the TracStar LDP and Zoom 88 large-bore guide catheters is feasible and safe in achieving intracranial access for neurointerventions in carefully selected patients.

Disclosures L. Lyons: None. M. Abouelleil: None. A. Restrepo Orozco: None. L. Verhey: None. J. Tsai: 2; C; Cerenovus, Medtronic. P. Mazaris: 2; C; Stryker. J. Singer: 2; C; Medtronic, Cerenovus, Stryker, Pneumbra, Nico.

\section{E-083 SAFETY AND EFFICACY OF XACT STENT IN TANDEM INTERNAL CAROTID ARTERY AND INTRACRANIAL LARGE VESSEL OCCLUSION}

M Oliver*, G Dawod, S Zaidi, M Jumaa. University of Toledo/Promedica, Toledo, $\mathrm{OH}$

10.1136/neurintsurg-2021-SNIS. 178

Introduction Endovascular stenting of the extracranial internal carotid artery (ICA) in the setting of tandem intracranial arterial occlusion (ICAO) is an area of ongoing research.

Objective We demonstrate the safety and efficacy of endovascular stenting of the ICA with tandem ICAO using the Xact stent.
Design This is a retrospective cohort analysis of all patients presenting to a single academic center with tandem ICA/ICAO who underwent mechanical thrombectomy from 2015-2020. Patients were included per AHA guidelines excluded if preprocedural angiography did not show tandem occlusions.

Main outcomes and measures Baseline variables included age, baseline National Institute of Health Stroke Scale (NIHSS), baseline Alberta Stroke Program Early CT Score (ASPECTS), site of intracranial occlusion, treatment techniques and time efficiencies, and thrombolysis in cerebral infarction score. Outcome measures included modified Rankin scale (mRs) at 90 days, median infarct volume, stent complications, stent restenosis rate, recurrent stroke at 30 days and symptomatic intracerebral hemorrhage (sICH).

Results A total of 67 patients with symptomatic angiographically-confirmed tandem ICA/ICAO were identified. The median patient age was 66, baseline median NIHSS was 16.2, Mean ASPECTS 8.1, ICAO location was the M1 segment of middle cerebral artery (MCA) in 39\% of patients, internal carotid artery terminus in 37\%, M2 segment of MCA in $23 \%$ and M3 segment of MCA in $1.6 \%$ of patients. Successful reperfusion of TICI 2B-3 was achieved in 91\% of patients. Favorable mRS of $0-2$ was achieved in 52\% of cases, mortality was $19 \%$, and median final infarct volume was $30.9 \mathrm{~mL}$. The rate of stent complications occurred in 3\% of patients, most common being partial in stent thrombosis extending intraluminally. Rate of sICH was 4\%, recurrent stroke occurred in $5 \%$ of patients within 30 days, and in-stent stenosis $>50 \%$ happened in $16 \%$ at 18 months. No patients required additional interventions.

Conclusions The Xact carotid stent is safe and efficacious in the treatment of tandem ICA/ICAO lesions. Larger prospective trials are needed to help confirm our retrospective findings.

Disclosures M. Oliver: None. G. Dawod: None. S. Zaidi: None. M. Jumaa: None.

\section{E-084 NICARDIPINE VERSUS CLEVIDIPINE FOR POST MECHANICAL THROMBECTOMY BLOOD PRESSURE MANAGEMENT IN PATIENTS WITH ISCHEMIC STROKE DUE TO ISOLATED MIDDLE CEREBRAL ARTERY OCCLUSION}

${ }^{1} \mathrm{M}$ Oliver*, ${ }^{1} \mathrm{~J}$ Shawwer, ${ }^{1} \mathrm{H}$ Salahuddin, ${ }^{2} \mathrm{~S}$ Desai, ${ }^{1} \mathrm{~S}$ Zaidi, ${ }^{1} \mathrm{M}$ Jumaa. ${ }^{1}$ University of Toledo/ Promedica, Toledo, $\mathrm{OH}^{2}{ }^{2}$ Barrow Neurological Institute, Phoenix, AZ

\subsection{6/neurintsurg-2021-SNIS. 179}

Objective Intensive blood pressure (BP) management after mechanical thrombectomy (MT) may be beneficial in patients with acute anterior circulation (AC) Ischemic stroke (IS) due to large vessel occlusion (LVO). We sought to evaluate the efficacy of Nicardepine (NCR) vs. Clevidipine (CLV) in IS patients with LVO who underwent successful MT.

Methods With IRB approval, we retrospectively collected data on consecutive patients with isolated MCA M1 occlusion who underwent successful MT (TICI score of 2B or 3). Patient demographics, baseline characteristics, time efficiencies, procedural data, incidence of hemorrhagic transformation (HI) and symptomatic ICH per ECASS II criteria on $24 \mathrm{H}$ head CT, and clinical outcomes were recorded. We also recorded duration from recanalization to optimal BP control (ROBP), IV drip utilized (NCR vs. CLV), number of SBP excursions, and total duration of time outside the target SBP in first $24 \mathrm{H}$. 